\title{
Molecular characterization of bovine leukemia virus reveals existence of genotype 4 in Chinese dairy cattle
}

\author{
Yi Yang ${ }^{1,2+}$, Lina Chen ${ }^{1 \dagger}$, Maoli Dong ${ }^{1}$, Wenjiang Huang ${ }^{1,3}$, Xiaoli Hao ${ }^{1}$, Yalan Peng ${ }^{1}$, Zaicheng Gong ${ }^{1}$, Aijian Qin ${ }^{1,2}$, \\ Shaobin Shang ${ }^{1,2^{*}}$ and Zhangping Yang ${ }^{2,4^{*}}$
}

\begin{abstract}
Bovine leukemia virus (BLV) causes enzootic bovine leucosis and is widely spread worldwide, except several European countries, Australia and New Zealand. Although BLV is highly prevalent in China, information about the genetic diversity and evolutionary dynamics of BLV among Chinese dairy herds is still lacking. To determine the genetic variability of BLV, 219 cows from four cities of Ningxia province of China were screened for BLV infection by fluorescence resonance energy transfer (FRET)-PCR and sequencing, 16 selected positive samples were subjected to molecular characterization. Phylogenetic analysis using the neighbor-joining (NJ) method on complete sequences of envelope (env) gene of BLV obtained from China and those available in GenBank (representing BLV genotypes 1-10) revealed that those Chinese strains belonged to genotypes 4 and 6. Totally, 23 mutations were identified and 16 of them were determined to be unique mutations among Chinese strains. Alignment of the deduced amino acid sequences demonstrated six mutations in glycoprotein 51 (gp51) and three mutations in glycoprotein 30 (gp30) located in the identified neutralizing domain (ND), CD8 ${ }^{+}$T cell epitope, E-epitope, B-epitope, gp51N12 and cytoplasmic domain of transmembrane protein. This study reported for the first time the BLV genotype 4 in China, and further studies are warranted to compare its immunogenicity and pathogenicity with other BLV genotypes.
\end{abstract}

Keywords: Bovine leukemia virus, Molecular characterization, Envelope gene, Genetic diversity

\section{Main text}

Bovine leukemia virus (BLV) is the causative agent of enzootic bovine leucosis (EBL), and approximately $30 \%$ of infected cattle develop persistent lymphocytosis (PL) while a small percentage of infected cattle die from malignant lymphoma. In recent years, a variety of methods have been applied for BLV genotyping [1-3]. Due to the biological functions, most of studies have primarily focused on the env gene. To date, at least 11 genotypes of BLV have been described based on the genetic polymorphism of the env gene [4, 5]. Previous studies demonstrated that

\footnotetext{
* Correspondence: shaobinshang@yzu.edu.cn; yzp@yzu.edu.cn

${ }^{\dagger}$ Yi Yang and Lina Chen contributed equally to this work.

${ }^{1}$ Jiangsu Co-innovation Center for Prevention and Control of Important Animal Infectious Diseases and Zoonoses; College of Veterinary Medicine, Yangzhou University, Yangzhou 225009, Jiangsu, China

${ }^{2}$ International Corporation Laboratory of Agriculture and Agricultural Products Safety, Yangzhou University, Yangzhou 225009, Jiangsu, China Full list of author information is available at the end of the article
}

BLV was widely spread among dairy herds in China, and genotypes 6, 10 and 11 existed in Chinese dairy or yak herds $[6,7]$.

From November 2018 to January 2019, bovine whole blood samples $(n=219)$ from four cities (Shizuishan, Yinchuan, Wuzhong and Zhongwei) of Ningxia province were submitted to Yangzhou University College of Veterinary Medicine for BLV identification. All samples were freshly collected in ethylenediaminetetraacetic acid (EDTA) blood collection tubes by Center for Animal Disease Control and Prevention of Ningxia province, and delivered on ice with next-day delivery. DNA was extracted from whole blood samples using commercial kit as previously described [8]. The FRET-qPCR targeting BLV pol gene (forward primer $=5^{\prime}$-CCTCAATTCCCTTT AAACTAGAACG-3'; reverse primer $=5^{\prime}$ - ATGGGC TTTGTAAGAGCATTTGTA-3'; anchor probe $=5^{\prime}$ GACGGGCCAGGCAATAATCCAGT-(6-FAM)-3';

(c) The Author(s). 2019 Open Access This article is distributed under the terms of the Creative Commons Attribution 4.0 International License (http://creativecommons.org/licenses/by/4.0/), which permits unrestricted use, distribution, and 
reporter probe $=5^{\prime}-($ LCRed640 $)$-TTCCCGGTACGG AAACCAAATGG-phosphate-3') was performed following the protocol previous described [9].In total, forty samples were identified to be positive from 219 whole blood samples. Copy numbers of BLV in positive cows ranged from 20 copies/ $\mathrm{ml}$ of whole blood to 362,936 copies/ ml of whole blood (mean 19,134 copies/ $\mathrm{ml}$ of whole blood and median 35 copies/ ml of whole blood) (Table 1), and those above 130 copies/ $\mathrm{ml}(n=16)$ were further identified for genotyping based on the diversity of env gene.

Partial sequence of BLV env gene were amplified with an in-house regular PCR [6]. Amplicons were gel purified with the QIAquick Gel Extraction Kit and sequenced with both forward and reverse primers at the GenScript Biotech Corp. (Nanjing, China). Sequence data (PCR products based on the forward and reverse primers) obtained in this study were assembled with DNASTAR Lasergen 15.2 (DNASTAR Inc., Madison, WI) and aligned using CLUSTAL W in MEGA 7.0 (MEGA, Pennsylvania State University, University Park) along with those of BLV strains found on GenBank from around the world. A neighbor-joining (NJ) phylogenetic tree was constructed using the Tamura-Nei model $[3,10]$ and the robustness of clusters was assessed by bootstrapping 1,000 replicates. Maximum-likelihood (ML) phylogenetic analysis was performed to confirm the results (Additional file 1 Figure S1).

Those sequences obtained in this study that were not identical to each other were submitted to
GenBank with the GenBank accession numbers: MK820044 and MK840875-MK840880. A neighborjoining phylogenetic tree based on the env gene complete sequences $(1,548 \mathrm{bp})$ of the Chinese strains and 37 reference strains representing BLV genotypes 1 to 10 from 14 countries demonstrated that three Chinese strains (MK820044, MK840877 and MK840879) belonged to genotype 4 and the remaining four (MK840875, MK840876, MK840878 and MK840880) belonged to genotype 6 (Fig. 1).

For those three Chinese strains clustered into BLV genotype 4, the mean distance of the env nucleotides and the deduced amino acid (AA) were $0.003 \pm 0.001$ and $0.008 \pm 0.004$ between these strains, respectively (Table 2). Compared with the BLV strains obtained from GenBank representing BLV genotypes 1 to 10 , the Chinese strains had between $0.014 \pm 0.002$ and $0.038 \pm 0.005$ nucleotide distance. Similarly, the Chinese strains had between $0.012 \pm 0.003$ and $0.037 \pm$ 0.008 deduced AA distance compared with these reference strains (Table 2). The distance of nucleotide and deduced AA indicated that these three Chinese BLV strains were mostly similar to genotype 4 but distinct from genotype 5 .

For the remaining four strains clustered into BLV genotype 6, the mean distance of the env nucleotides and the deduced AA were $0.004 \pm 0.001$ and $0.002 \pm$ 0.002 , respectively (Table 2). Compared with the BLV strains obtained from GenBank, the Chinese strains had nucleotide distance between $0.020 \pm 0.003$ and $0.046 \pm 0.005$. Similarly, the Chinese strains had

Table 1 Test result of BLV infection in Ningxia province by FRET-qPCR

\begin{tabular}{|c|c|c|c|c|c|}
\hline City & County & $\begin{array}{l}\text { BLV positivity by } \\
\text { FRET-qPCR }\end{array}$ & $\begin{array}{l}\text { Copy number range (per } \mathrm{ml} \text { of } \\
\text { whole blood) }\end{array}$ & $\begin{array}{l}\text { Copy number mean (per } \mathrm{ml} \text { of } \\
\text { whole blood) }\end{array}$ & $\begin{array}{l}\text { Copy number median (per } \mathrm{ml} \text { of } \\
\text { whole blood) }\end{array}$ \\
\hline \multirow[t]{4}{*}{ Shizuishan } & Pingluo & $2 / 17(11.76 \%)$ & $1829-6865$ & 4347 & 4347 \\
\hline & Huinong & $3 / 21$ (14.29\%) & 20-130 & 57 & 20 \\
\hline & Dawu & 4/14 (28.57\%) & 20-130 & 79 & 83 \\
\hline & Total & 9/52 (17.31\%) & $20-6865$ & 1020 & 130 \\
\hline \multirow[t]{6}{*}{ Yinchuan } & Jinfeng & $3 / 20(15.00 \%)$ & $20-362,936$ & 120,992 & 20 \\
\hline & Yongning & 2/17 (11.76\%) & 35 & 35 & 35 \\
\hline & Xingqing & $10 / 20(50.00 \%)$ & $20-362,936$ & 37,205 & 83 \\
\hline & Xixia & 0/21 (0.00\%) & N/A & N/A & N/A \\
\hline & Lingwu & $6 / 12$ (50.00\%) & 20-130 & 41 & 20 \\
\hline & Total & $21 / 90$ (23.33\%) & $20-362,936$ & 35,016 & 35 \\
\hline \multirow[t]{4}{*}{ Wuzhong } & Litong & 4/19 (21.05\%) & 130-6865 & 5181 & 6865 \\
\hline & Yanchi & 2/18 (11.11\%) & 20 & 20 & 20 \\
\hline & Qingtongxia & 0/21 (0.00\%) & N/A & N/A & N/A \\
\hline & Total & 6/58 (10.34\%) & $20-6865$ & 3461 & 3498 \\
\hline Zhongwei & Zhongning & 4/19 (21.05\%) & 20 & 20 & 20 \\
\hline Total & & $40 / 219(18.26 \%)$ & $20-362,936$ & 19,134 & 35 \\
\hline
\end{tabular}




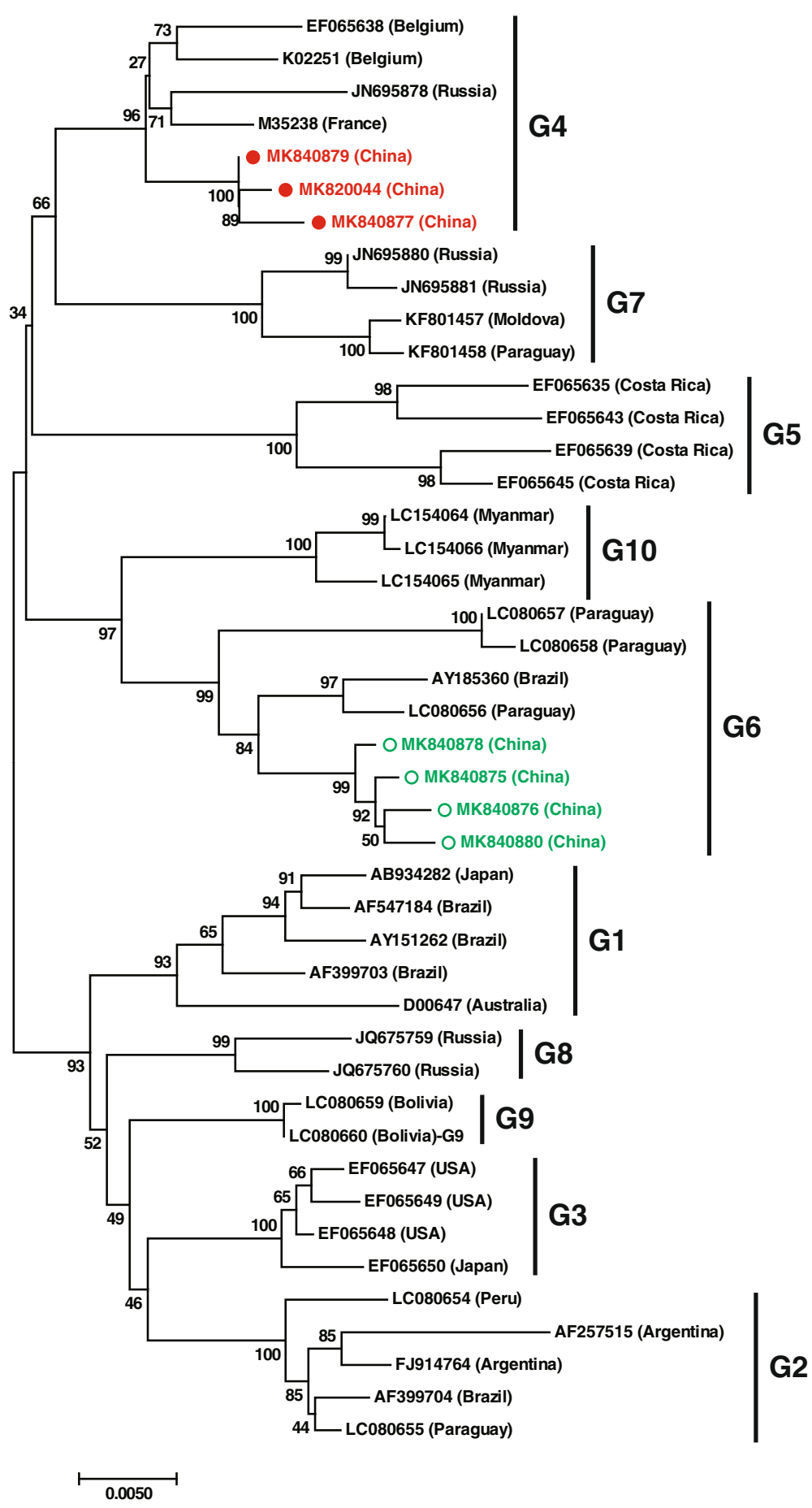

Fig. 1 Neighbor-joining phylogenetic tree based on BLV env gene complete sequences (1548 bp) from China and around the world. Strains identified in our study in China are identified with filled circles (•) for genotype 4 (in red) and open circles (o) for genotype 6 (in green). Genotypes shown on the right are according to Yang et al. [6]. The numbers at the branches show bootstrap support (1000 replicates). The bar at the bottom of the figure denotes distance

between $0.008 \pm 0.003$ and $0.037 \pm 0.008$ deduced AA distance compared with those reference strains (Table 2). The distance of nucleotide and deduced AA indicated that the three Chinese BLV strains were mostly similar to genotype 6 but distinct from genotype 5 .

Although 10 genotypes of BLV have been discovered around the world, there is little information on 


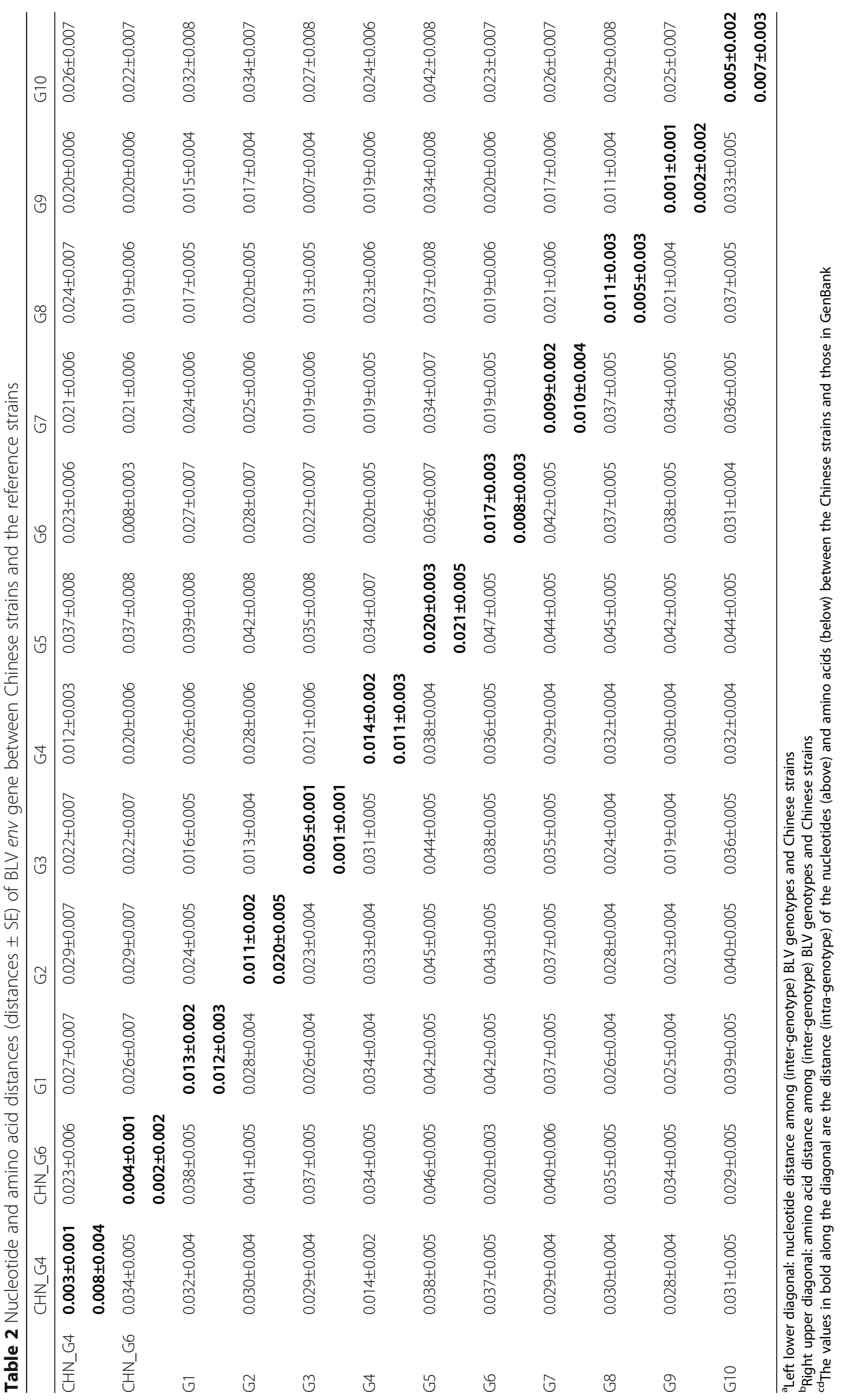




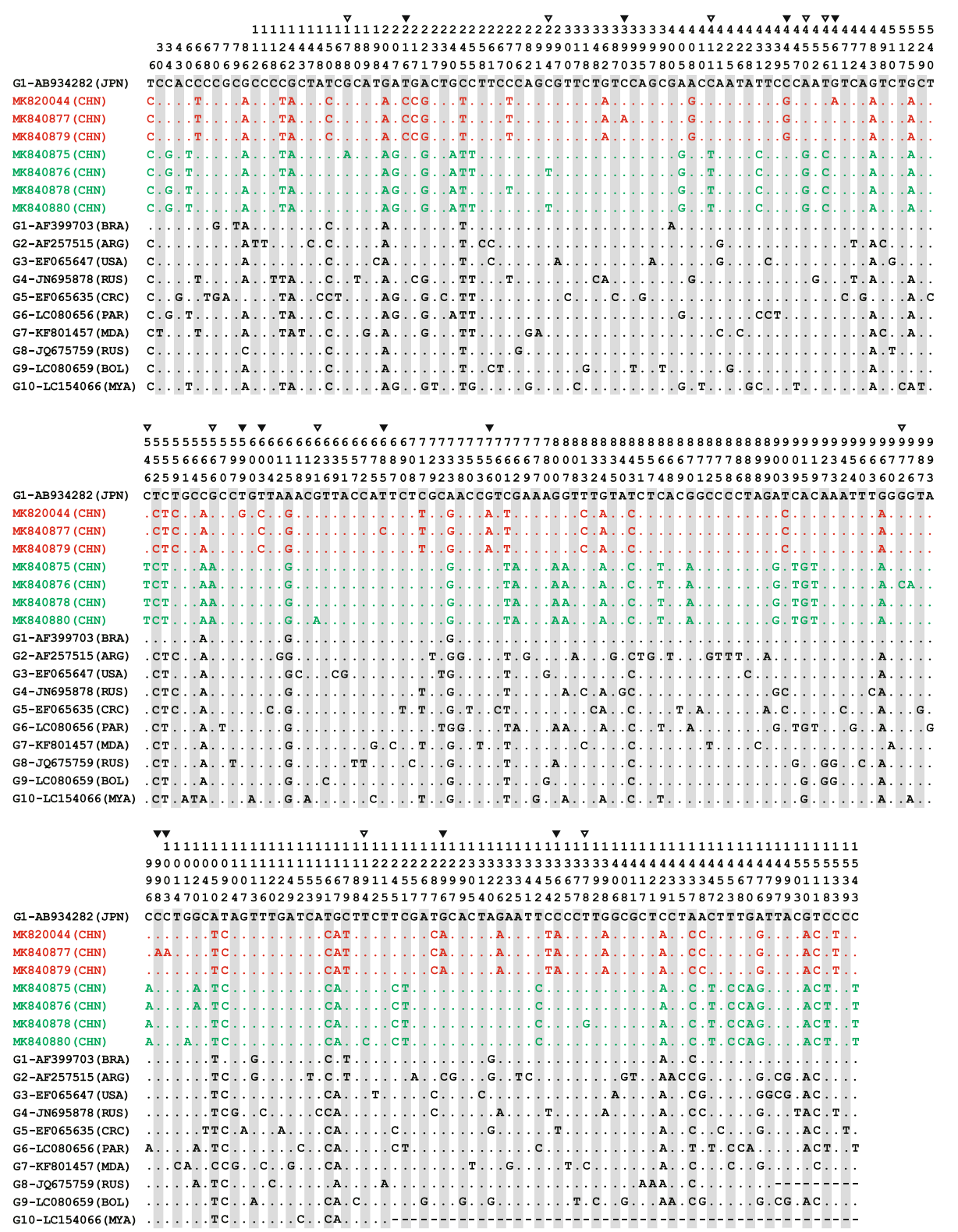

Fig. 2 Alignment of full-length of BLV env gene nucleotide sequences (1548 bp) between sequences obtained in this study together with 10 reference sequences obtained in the GenBank database. Strains identified in this study are in red (cluster into genotype 4) and green (cluster into genotype 6). Numbers above the sequences are nucleotide number indicated by the env gene of AB934282. The countries of the strains are marked with abbreviations in parentheses to the right of the GenBank accession numbers. Dots indicate nucleotides identical to the reference sequences. The mark above the square frames indicate mutations for our isolates of BLV genotype $4(\boldsymbol{\nabla})$ and genotype $6(\nabla)$. The BLV reference strains from GenBank have accession numbers AF933703 (G1), AF257515 (G2), EF065647 (G3), JN695878 (G4), EF065635 (G5), LC080656 (G6), KF801457 (G7), JQ675759 (G8), LC080659 (G9), and LC154066 (G10). The seven Chinese strains from this study have the accession numbers MK820044 and MK840875-MK840880. JPN = Japan; CHN = China; BRA = Brazil; ARG = Argentina; USA = United States of America; RUS = Russia; $\mathrm{CRC}=$ Costa Rica; $\mathrm{PAR}=$ Paraguay; $\mathrm{MDA}=$ Moldova; $\mathrm{BOL}=$ Bolivia; $\mathrm{MYA}=$ Myanmar

genetic diversity of BLV among Chinese dairy herds [11], until BLV genotype 6 was firstly identified in Yancheng, Shanghai, Yangzhou, Bengbu and Tianjin in 2019 [6]. The present study revealed the existence of BLV genotype 4 in China for the first time. When compared with the reference sequences representing all 10 BLV genotypes deposited in GenBank, we found that our Chinese isolates had a total of 23 mutations in complete env gene. Among them, fourteen were synonymous mutations (T216C, C294T, C390A, C411T, A450G, T456C, C546T, T603C, G621A, G756A, G972C, T1194C, G1296A 
and $\mathrm{C} 1362 \mathrm{~A}$ ) and the remaining nine were nonsynonymous mutations (G178A, C445G, G461A, G566A, T599G, T685C, C998A, C1003A and T1378G). Interestingly, the Chinese strains of BLV genotype 4 and genotype 6 have 12 and 11 mutations, respectively on the env gene that was not shared between the two groups (Fig. 2). The distance between BLV genotype 4 and 6 was $0.036 \pm$ 0.005 (nucleotide) and $0.020 \pm 0.005$ (AA). When compared with the 10 reference sequences and all Chinese sequences available in the GenBank database, 16 of the mutations (G178A, T216C, C390A, C445G, G461A, T599G, T603C, T685C, G756A, G972C, C998A, C1003A, T1194C, G1296A, C1362A and $\mathrm{T} 1378 \mathrm{G}$ ) were identified as unique mutations (Additional file 2 Figure S2-A and S2-B).

Among all the nonsynonymous mutations, seven of them (G178A, G461A, T599G, T685C, C998A, C1003A and T1378G) were observed in single isolate and the remaining two (C445G and G566A) were existed in three or four isolates. Alignment of deduced amino acid sequences demonstrated that six mutations (D60N, L149 V, G154E, R189N, L200R and $\mathrm{Y} 229 \mathrm{H}$ ) were in glycoprotein 51 (gp51) and three mutations (T333 N, L335 M and L460 V) were in glycoprotein 30 (gp30), distributing respectively in the neutralizing domain $2, \mathrm{CD}^{+} \mathrm{T}$ cell epitope, E-epitope, B-epitope, gp51N12 and cytoplasmic domain of the transmembrane protein [12-14] (Fig. 3).

This study investigated the prevalence and genetic variability of BLV and identified the BLV genotype 4 in China for the first time. Together with our previous study [6] and studies conducted by Wang and Yu in 2018 and 2019 [5, 7], BLV genotypes 1, 4, 6 and 10 were present in dairy cattle or yaks in China. BLV genotype 4 is the second most common genotype prevalent worldwide and was identified in Mongolia in 2016 [2]. The cow trade between China and Mongolia might contributed to the spread of BLV between the two countries. This study will help us to better understand the genetic diversity of BLV in China. However, further studies are needed to define the immunogenicity and pathogenicity between different genotypes of BLV.

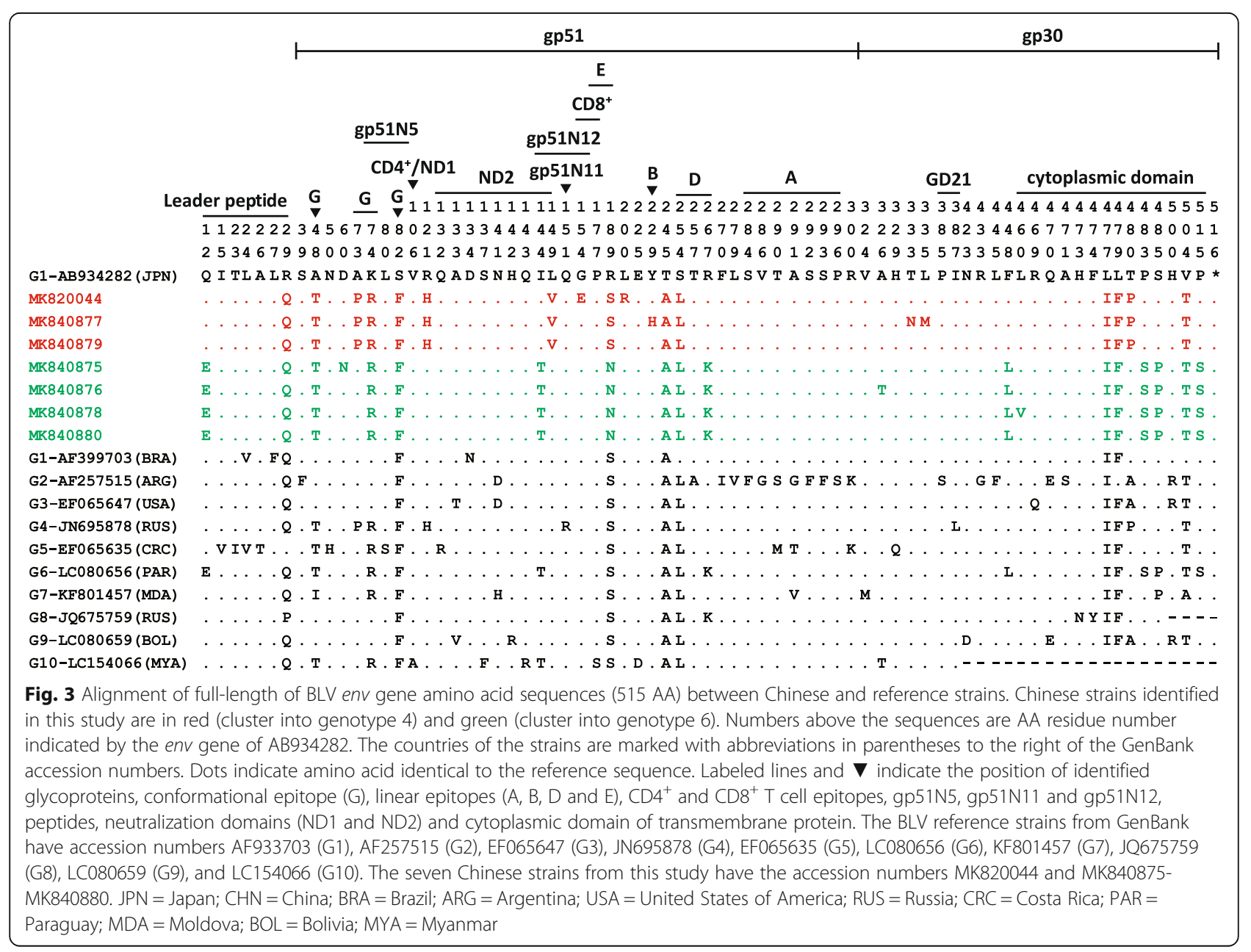




\section{Additional files}

Additional file 1: Figure S1. Maximum-likelihood phylogenetic tree based on BLV env gene complete sequences (1548 bp) from China and around the world. Strains identified in our study in China are identified with filled circles $(\bullet)$ for genotype 4 (in red) and open circles (o) for genotype 6 (in green). Genotypes shown on the right are according to Yang et al. [6]. The numbers at the branches show bootstrap support (1000 replicates). The bar at the bottom of the figure denotes distance. (Al 1649 kb)

Additional file 2 Figure S2. Alignment of full-length of BLV env gene nucleotide sequences (S2-A: 6-769 bp; S2-B: 777-1543 bp) between sequences obtained in this study together with 10 reference sequences and all Chinese sequences available in the GenBank database. Strains identified in this study are in red (cluster into genotype 4) and green (cluster into genotype 6). Numbers above the sequences are nucleotide number indicated by the env gene of AB934282. The countries of the strains are marked with abbreviations in parentheses to the right of the GenBank accession numbers. Dots indicate nucleotides identical to the reference sequences. The mark above the square frames indicate unique mutations for our isolates of BLV genotype $4(\boldsymbol{\nabla})$ and genotype $6(\nabla)$. The BLV reference strains from GenBank have accession numbers AF933703 (G1), AF257515 (G2), EF065647 (G3), JN695878 (G4), EF065635 (G5), LC080656 (G6), KF801457 (G7), JQ675759 (G8), LC080659 (G9), and LC154066 (G10). The Chinese sequences available in the GenBank database have the accession numbers: MH040198-MH040203, MH040205, MH040207-MH040209, MF574053-MF574068. The seven Chinese strains from this study have the accession numbers MK820044 and MK840875MK840880. JPN = Japan; $C H N=$ China; $B R A=$ Brazil; $A R G=$ Argentina; USA = United States of America; RUS = Russia; $C R C=$ Costa Rica; PAR = Paraguay; MDA = Moldova; $\mathrm{BOL}=$ Bolivia; $\mathrm{MYA}=$ Myanmar. (ZIP 5157 kb)

\section{Abbreviations}

AA: amino acid; ARG: Argentina; BLV: Bovine leukemia virus; BOL: Bolivia; BRA: Brazil; CHN: China; CRC: Costa Rica; EBL: Enzootic bovine leucosis; EDTA: Ethylenediaminetetraacetic acid; Env: envelope; FRET: Fluorescence resonance energy transfer; gp30: glycoprotein 30; gp51: glycoprotein 51; JPN: Japan; MDA: Moldova; ML: Maximum likelihood; MYA: Myanmar; ND: Neutralizing domain; NJ: Neighbor-joining; PAR: Paraguay; PL: Persistent Iymphocytosis; RUS: Russia; USA: United States of America

\section{Acknowledgements}

We thank Jun Ma and Haining Zhou (Center for Animal Disease Control and Prevention of Ningxia province) for dairy whole blood sample collection.

\section{Authors' contributions}

$S S, Z Y, Y Y, W H$ and $A Q$ conceived and designed the study. LC, MD, XH, YP and $Z G$ performed the experiments. $Y Y$ analyzed the epidemiological data. YY and SS wrote the manuscript. All authors read and approved the final version of the manuscript.

\section{Funding}

This study was supported by China Postdoctoral Science Foundation (2019 M650126), Basic Research Program of Jiangsu Province and the Priority Academic Program Development of Jiangsu Higher Education Institutions (PAPD).

\section{Availability of data and materials}

The sequences of full-length envelope gene generated in this study have been deposited in GenBank under the accession numbers MK820044 and MK840875-MK840880.

\section{Ethics approval and consent to participate}

Protocols for the collection of samples in this study were reviewed and approved by the Institutional Animal Care and Use Committee of Yangzhou University College of Veterinary Medicine.

\section{Competing interests}

The authors declare that they have no competing interests.

\section{Author details}

${ }^{1}$ Jiangsu Co-innovation Center for Prevention and Control of Important Animal Infectious Diseases and Zoonoses; College of Veterinary Medicine, Yangzhou University, Yangzhou 225009, Jiangsu, China. ${ }^{2}$ International Corporation Laboratory of Agriculture and Agricultural Products Safety, Yangzhou University, Yangzhou 225009, Jiangsu, China. ${ }^{3}$ Victor Pharmaceutical Company of Limited Liability, Zhenjiang 212132, Jiangsu, China. ${ }^{4}$ College of Animal Science and Technology, Yangzhou University, Yangzhou 225009, Jiangsu, China.

Received: 12 June 2019 Accepted: 23 July 2019

Published online: 27 August 2019

\section{References}

1. Felmer R, Muñoz G, Zúñiga J, Recabal M. Molecular analysis of a 444 bp fragment of the bovine leukaemia virus gp51 env gene reveals a high frequency of non-silent point mutations and suggests the presence of two subgroups of BLV in Chile. Vet Microbiol. 2005;108:39-47.

2. Ochirkhuu N, Konnai S, Odbileg R, Nishimori A, Okagawa T, Murata S, et al. Detection of bovine leukemia virus and identification of its genotype in Mongolian cattle. Arch Virol. 2016;161:985-91.

3. Polat M, Ohno A, Takeshima SN, Kim J, Kikuya M, Matsumoto Y, et al. Detection and molecular characterization of bovine leukemia virus in Philippine cattle. Arch Virol. 2015;160:285-96.

4. Lee E, Kim EJ, Ratthanophart J, Vitoonpong R, Kim BH, Cho IS, et al. Molecular epidemiological and serological studies of bovine leukemia virus (BLV) infection in Thailand cattle. Infect Genet Evol. 2016:41:245-54.

5. Yu C, Wang X, Zhou Y, Wang Y, Zhang X, Zheng Y. Genotyping bovine leukemia virus in dairy cattle of Heilongjiang, northeastern China. BMC Vet Res. 2019;15:179.

6. Yang Y, Chu S, Shang S, Yang Z, Wang C. Genotyping and single nucleotide polymorphism analysis of bovine leukemia virus in Chinese dairy cattle. J Dairy Sci. 2019;102:3469-73.

7. Wang M, Wang Y, Baloch AR, Pan Y, Xu F, Tian L, et al. Molecular epidemiology and characterization of bovine leukemia virus in domestic yaks (Bos grunniens) on the Qinghai-Tibet plateau, China. Arch Virol. 2018;163:659-70.

8. Yang Y, Yang Z, Kelly P, Li J, Ren Y, Wang C. Borrelia miyamotoi sensu lato in Père David deer and Haemaphysalis longicornis ticks. Emerg Infect Dis. 2018;24:928-31.

9. Yang Y, Fan W, Mao Y, Yang Z, Lu G, Zhang R, et al. Bovine leukemia virus infection in cattle of China: association with reduced milk production and increased somatic cell score. J Dairy Sci. 2016;99:3688-97.

10. Rola-Łuszczak M, Pluta A, Olech M, Donnik I, Petropavlovskiy M, Gerilovych A, et al. The molecular characterization of bovine leukaemia virus isolates from Eastern Europe and Siberia and its impact on phylogeny. PLoS One. 2013;8:e58705

11. Polat M, Takeshima SN, Aida Y. Epidemiology and genetic diversity of bovine leukemia virus. Virol J. 2017;14:209.

12. Moratorio G, Fischer S, Bianchi S, Tomé L, Rama G, Obal G, et al. A detailed molecular analysis of complete bovine leukemia virus genomes isolated from B-cell lymphosarcomas. Vet Res. 2013;44:19.

13. Polat M, Takeshima SN, Hosomichi K, Kim J, Miyasaka T, Yamada K, et al. A new genotype of bovine leukemia virus in South America identified by NGS-based whole genome sequencing and molecular evolutionary genetic analysis. Retrovirology. 2016;13:4.

14. Bai L, Takeshima SN, Isogai E, Kohara J, Aida Y. Novel CD8(+) cytotoxic T cell epitopes in bovine leukemia virus with cattle. Vaccine. 2015;33:7194-202.

\section{Publisher's Note}

Springer Nature remains neutral with regard to jurisdictional claims in published maps and institutional affiliations.

\section{Consent for publication}

Not applicable. 